\title{
Exciton binding energies and photo-induced tunnelling of carriers in (Ga,In)As-GaAs heterostructures grown with built-in piezoelectric field
}

\author{
P. BIGENWALD, P. BORING, K.J. MOORE*, B. GIL and K. WOODBRIDGE**
}

Groupe d'Etudes des Semiconducteurs, Université de Montpellier II, case coumier 074, 34095 Montpellier cedex 5, France

${ }^{*}$ The Manchester Metropolitan University, Department of Mathematics and Physics, John Dalton

Building, Chester Street, Manchester M1 5GD, U.K.

${ }^{* *}$ University College London, Department of Electronic and Electrical Engineering, London WC1E 7JE, U.K.

\begin{abstract}
We compare the binding energy of interacting electron and hole pairs in double quantum wells with and without internal piezo-electric fields. We show that the exciton binding is less sensitive to the piezo electric field than the oscillator strength. This allows many body-effects and bandgap renormalization to be easily produced in strained-layer quantum wells with internal built-in piezo-electric fields, under photo excitation. Our observation was made at low temperature by comparing the behaviour of Ga. $a_{0 .} \ln _{0.08 A s-G a A s}$ strained layer single and double quantum wells grown along the (001) and (111) directions when the densities of photoinjected carriers are tuned over several decades. Comparison between experimental data and the results of a Hartree calculation including the space charge effects reveals that manybody interactions are efficiently photo-induced in the (111)-grown samples. Moreover, tunnelling of the two first excited heavy-hole levels can be stimulated for moderate carrier densities making such structure promissive for realising self electrooptic effect device (SEED) modulators.
\end{abstract}

\section{I-Introduction}

Piezoelectricity evidenced by $\mathrm{P}$. and J. Curie in 1880 has lead to numerous industrial applications which appeared during World War I (acoustic detection of submarines for instance). This property appears in crystal not having inversion symmetry. Any piezoelectric crystal polarises under application of an external strain field and reciprocally. The theory of the phenomenon which in turn results of the modification of the charge density in the deformed crystal is rather complicated and could only recently be accurately calculated ${ }^{1}$. Some of the various applications of piezoelectric crystal involve the mechanical resonance of piezoelectric devices (generation or filtering of frequencies, generation or reception of ultrasounds, sounds). Other applications don't take advantage of this resonance (stress sensors, sensors for electro-acoustic pressures). Ceramics (polycrystalline compounds with randomly oriented micro crystals), quartz and some of its analogues like berlinite for instance are the most world widely used compounds. When strained-layer 
compounds III-V or II-VI semiconductors are grown away from the (001) orientation, they may exhibit additional properties connected to the existence of strong built-in piezo-electric fields. Smith and Mailhiot, in a series of pioneering studies on superlattices 2 calculated the implication of the existence of such piezo-electric fields on conduction and valence states of semiconductor quantum wells and superlattices grown with strain along various directions. The semiconductor quantum well is simply a thin layer $(\sim 10 \mathrm{~nm})$ of a small gap semiconductor which, when sandwiched between two layers of higher band gap compounds acting as barriers, confines the carriers. Strained-layer quantum wells are generally obtained by coherent growth of lattice-mismatched compounds, using modern growth techniques. If growth of strained zinc-blende semiconductors occurs away from the three (001)-like directions, piezo-electric fields are created ${ }^{2}$. Experimental observation of the effects predicted by Smith and Mailhiot for semiconductor heterostructures has been demonstrated later in the literature ${ }^{3-12}$. The potential of strained layer heterostructures having such built-in piezo-electric fields could be valuable for making self-optic modulators by using an artfully designed quantum mechanical system like double quantum wells. This has stimulated us to compare the optical properties of (001)- and (111)B-grown (Ga, In)As-GaAs single and double quantum wells embedded in pin structures.

\section{II-Theory of the optical properties in the effective mass approximation}

Figure 1 displays the first electron and heavy-hole envelope functions calculated for both kinds of single quantum well structures, having a well width of 10 $\mathrm{nm}$ well, and $10 \%$ indium. For the (111) growth, substrate orientation is chosen such as that the pin field is one order of magnitude smaller than the piezo-electric field and opposite to it ${ }^{11}$. The envelope functions of the carriers are strongly spatially separated by the piezo-electric field.

\section{Figure 1}

Ga. $\ln _{0.1}$ As-GaAs strained-layer quantum wells in pin diodes

$$
E_{\text {pin }}=1.510^{4} \text { Volt } / \mathrm{cm}
$$
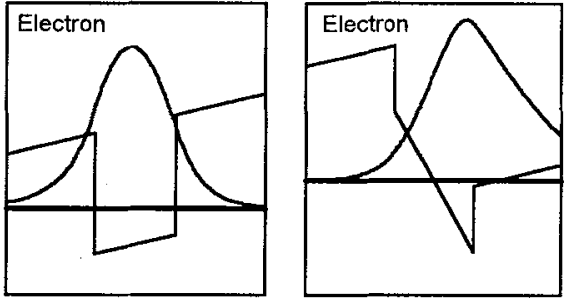

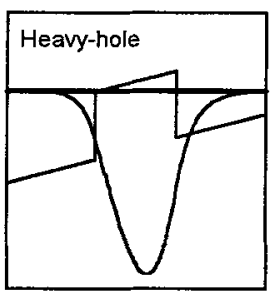

(001)-grown

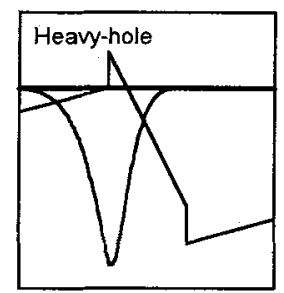

(111)B-grown

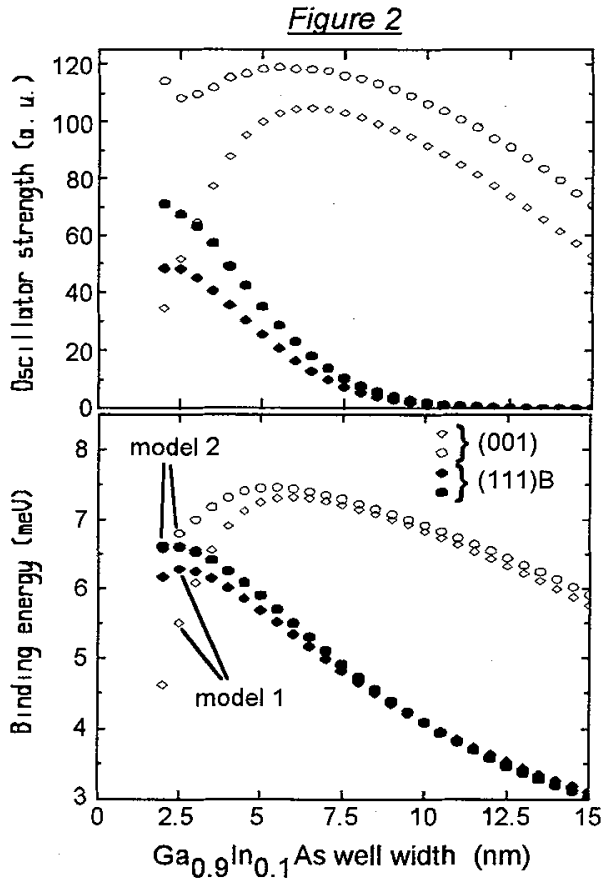


To quantify more concretely the difference between optical properties of such heterostructures in relation with the existence of the internal piezo-electric field, we have calculated the excitonic interaction in such samples in the context of the variational method, using two different trial functions. Model 1 uses a trial function $\Psi_{1}=\chi_{\theta}\left(z_{\theta}\right) \chi_{h}\left(z_{h}\right) \exp (-\rho / \lambda)$, while model 2 uses a more sophisticated trial function: $\Psi_{2}=\chi_{e}\left(z_{e}\right) \chi_{h}\left(z_{h}\right) \exp \left(-\sqrt{\rho^{2}+\left(z_{e}-z_{h}\right)^{2}} / \lambda\right)$, where $z_{e}, z_{h}, \rho$ are the electron, heavy-hole, and in-plane coordinates respectively. The variational parameter is $\lambda$. Figure 2 (lower part) summarises the results of the variational binding energies we calculate for the ground state heavy-hole exciton. We note that model 2 gives higher values for the excitonic interaction, due to the z-contribution to the exciton trial function. The oscillator strengths have also been calculated and are given in the upper part of the figure 2.

The reduction of the excitonic interaction in (111)B-grown wide wells, compared with (001)-grown ones is in straightforward relation with the strong spatial separation of the carriers under the effect of the internal piezo-electric field. This reduction is not as dramatic as what is obtained for oscillator strengths. The reason for this modest reduction lies in the long-range nature of the Coulomb interaction. In contrast to this, the oscillator strength is essentially linked to the spatial separation of the carrier by the piezo-electric field (middle range effect in samples designed such as that the pin field opposes to the piezo-electric field) via modification of the carrier wave functions. Although we compute rather comparable values for the exciton energies, the oscillator strengths display completely different behaviour with well-width. (111)B-grown wide wells should exhibit strong non linear optical properties, due to the existence of such internal piezoelectric field. Thus, we expect large values of the radiative lifetime ${ }^{13}$ (it is roughly speaking inversely proportional to the oscillator strength) and subsequent strong space charge effects for (111) B grown samples under laser illumination 11,12.

At this stage, we anticipate that advantage of the effect could be taken by optimisation of the sample design. Exalting the effect could be achieved by increasing the magnitude of the spatial separation of the electron-hole pair. This requires to grow wider wells or deeper ones (larger indium content). Unfortunately, the amount of elastic energy stored in the active part of the structure would increase, increasing the number of misfit dislocations. We believe that this would influence the reproducibility of the effect in a series of a priori identically-grown samples, and would prevent any industrial application. Besides this, including a thin (unstrained) GaAs barrier layer between two (Ga, In)As confining layers can also break the parity in a way sensibly equivalent with the growth of thick strained layer, or growth of indium-rich alloy layer. The advantage of this structure is, given a spatial separation of the electron-hole pair, to limit the number of potential dislocations compared to the single quantum well case. The results of the calculations of the effect characteristics of double quantum wells appear on figures 3 and 4. Comparing for instance figures 1 and 3 for identical alloy layer thicknesses (potential drop due to the piezo-electric field), it is obvious that inclusion of a thin GaAs barrier layer strongly increases the spatial separation of the electron-hole pair. Therefore the possibility to observe photo-induced screening of the piezo-electric field seems to be favoured by the double quantum well design. 
Figure 3

Ga $0.92^{\ln } 0.08$ As-GaAs strained-layer double quantum wells in pin diodes

$E_{\text {pin }}=1.510^{4} \mathrm{Volt} / \mathrm{cm}$
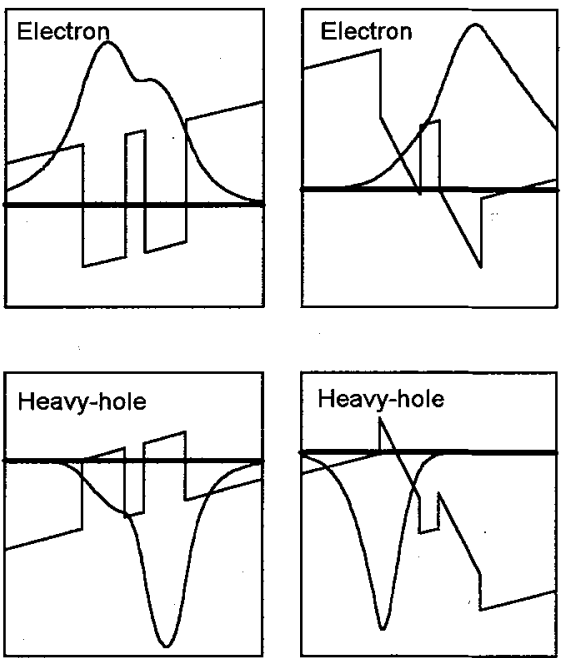

(001)-grown

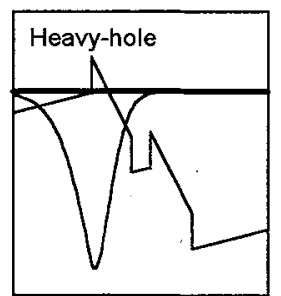

(111)B-grown
Figure 4

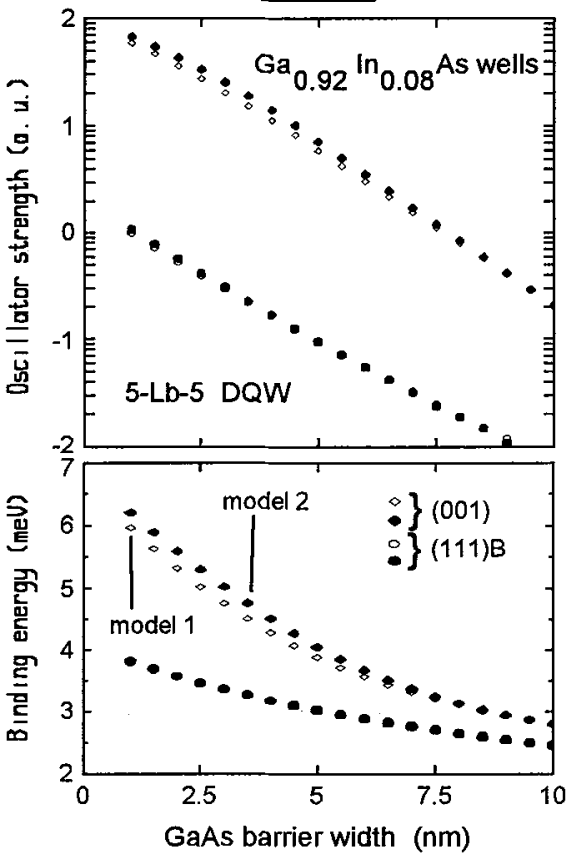

\section{III- Experimental results}

The structures we have investigated experimentally are strained-layer (Ga, In)As-GaAs single quantum wells grown along the (111) direction by Molecular Beam Epitaxy. To eliminate the deleterious influence of mobile charges on the piezoelectric effect ${ }^{2}$, we adopted the following designs: starting from a $n^{+}$-doped $\left(210^{18}\right.$ $\mathrm{cm}^{-3}$ ) GaAs substrate, we grew $0.5 \mu \mathrm{m}$ of non-intentionally doped GaAs ( $n_{a}-n_{d} \sim$ $10^{15} \mathrm{~cm}^{-3}$ ), followed by the active part of the sample (here a $9.3 \mathrm{~nm}$ Ga0.92 Ino.08As layer or two $5 \mathrm{~nm} \mathrm{Ga0.92}$ In $_{0.08}$ As layers with one thin $(2.5 \mathrm{~nm})$ internal $\mathrm{GaAs}$ barrier layer), $0.5 \mu \mathrm{m}$ of non-intentionally doped $\mathrm{GaAs}$ again, and 2 $\mu \mathrm{m}$ beryllium-doped $\left(p^{+} \sim 210^{18} \mathrm{~cm}^{-3}\right)$ GaAs. The Fermi level is pinned to the top of the valence/conduction band in the $p^{+} / n^{+}$regions respectively, giving a $1.510^{4}$ $\mathrm{V} / \mathrm{cm}$ nip field in the undoped region of the samples. Moreover, substrates were chosen to be (111)B-oriented so that the piezo-electric field which equals some 1.25 $10^{5} \mathrm{~V} / \mathrm{cm}$ in the strained layers, is opposite to the nip field.

Photoluminescence data taken on both (001)-grown and (111)B-grown samples have shown completely different behaviour: the photoluminescence energy does not significantly vary for the (001)-grown sample, whilst, for the (111)B-grown sample it exhibits a linear dependence with pump power at moderate power densities and saturates under high excitation conditions. Such blue-shift of some 25 meV has been observed in both single and double quantum wells grown along the (111) direction. 
Figure 5

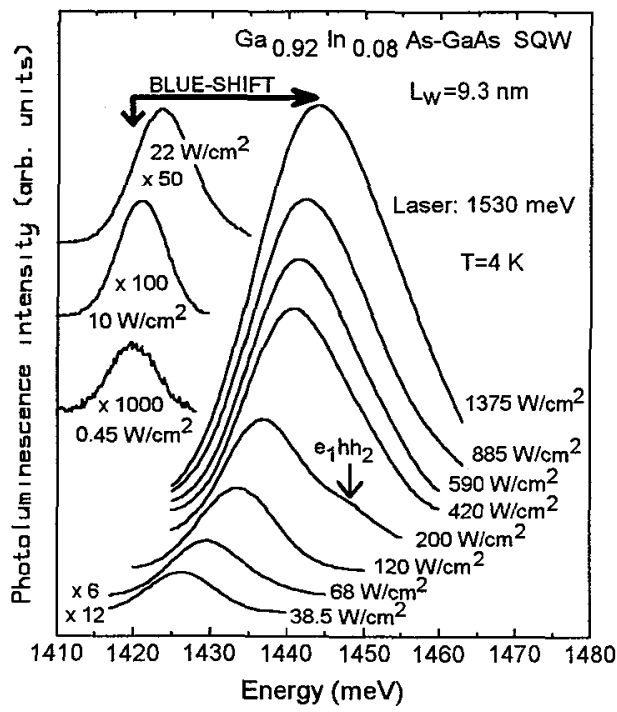

Figure 6

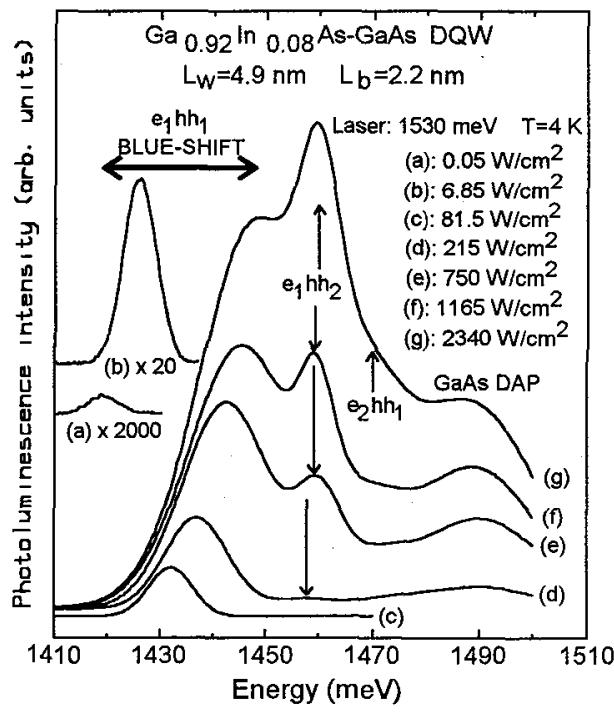

Figures 5 and 6 summarise the evolution of the photoluminescence signal for two (111)B-grown structures: a single and a double quantum well. We note that the main photoluminescence line broadens and that additional lines appear at high injection density. This we attribute to phase space filling by photo carriers. The observation of the additional features is linked to the crossing of the hole (electron) Fermi energy with the second heavy-hole (electron) subband at sufficient photo carrier densities. Figures 7 and 8 illustrate the evolution of the photoluminescence lines with excitation density.

Figure 7



Figure 8

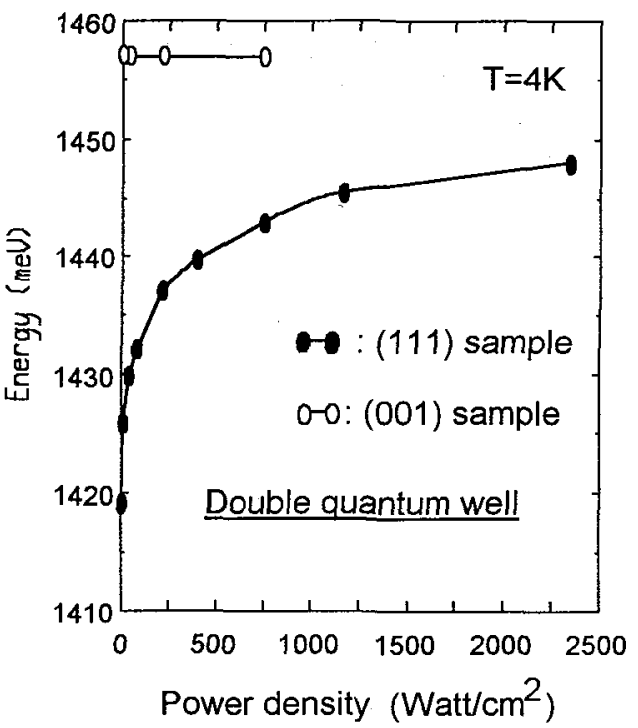




\section{IV-Theoretical calculation of the photo-induced screening of the piezo- electric field \\ To explain our observations we must consider a number of phenomena.} Firstly, the effect of space charge fields caused by the spatial separation of photoinjected electrons and holes. To calculate the magnitude of the space charge effect, and its implications on the PL with pump power, in the simplest approach, we have to solve self-consistently the equation:

$$
\left[-\frac{\hbar^{2}}{2} \frac{\partial}{\partial z}\left(\frac{1}{m_{i}^{*}(z)}\right) \frac{\partial}{\partial z}+V(z)+q[F(z)+\Phi(z)] z\right] \chi(z)=E \quad \chi(z)
$$

for each type of carrier. In this equation, $V$ is the potential line-up, $F$ is the total field without injected carriers, and $\Phi$ is the contribution of photo-injected electron-hole pairs. In the most general way, at $T \sim O K, \Phi$ is defined as:

$\Phi(z+d z)-\Phi(z)=e \sigma \int_{z}^{z+d z}\left[\sum_{m, i}^{k_{\perp}} \int_{0}^{(\sigma)} \alpha_{m, i}\left(k_{\perp}, \sigma\right) \chi_{h_{m, i}}^{2}\left(k_{\perp}, u\right) d k_{\perp}-\sum_{n, j} \int_{0}^{k_{\perp}(\sigma)} \beta_{n, j}\left(k_{\perp}, \sigma\right) \chi_{e_{n, j}}^{2}\left(k_{\perp}, u\right) d k_{\perp}\right] \frac{d u}{\varepsilon(u)}$

where the summations $\Sigma$ are extended over the $n$ electron $(e)$ and $m$ hole $(h)$ states of interest. Given an areal carrier density $\sigma$, one will fill one or several confined subbands up to an appropriate value of the in plane wave vector $k_{\perp}$. The indices $i$ and $j$ refer to the lifting of spin degeneracy away from $k_{\perp}=0$, and $\varepsilon(z)$ is the dielectric constant. We note that photo-induced screening of the piezo-electric field (included in F) will depend on the magnitude of $\Phi$. This quantity is at first order a linear function of the electron-hole pair density $\sigma$, but its local variations are in relation with the distribution of the carrier among the various states of interest. As a consequence the resulting effect is non-linear. This appears on figure 9 where we plot the average field $F+\Phi$ calculated in case of the single quantum well we studied as a function of the carrier density(full line). As an indication, the result given by the standard plane condensator model are also given (dashed line).

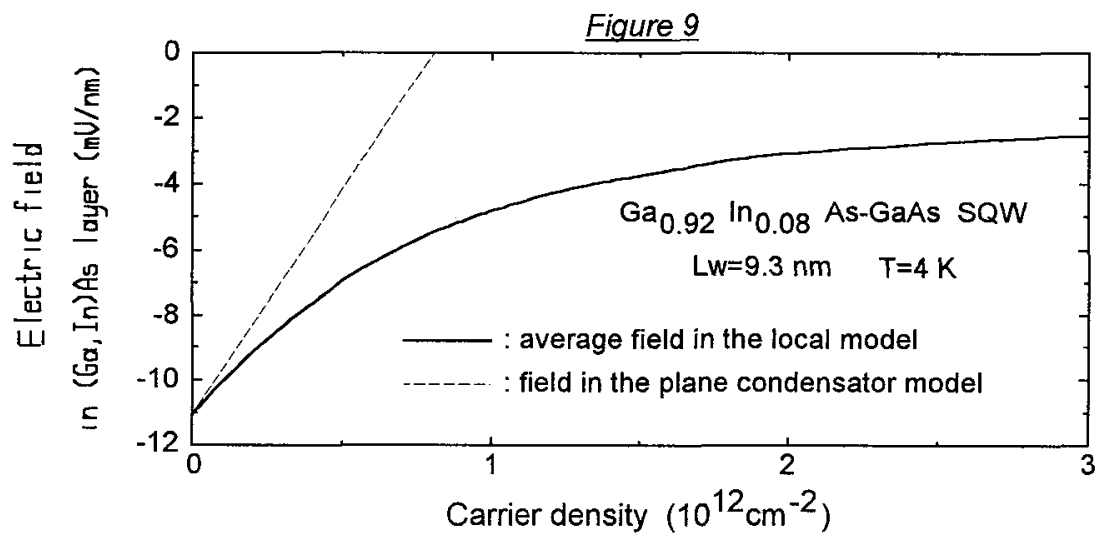


Figure 10 displays the result of the calculation for the samples of figures 5 and 6 . The area of the circles is proportional to the oscillator strength between conduction and valence envelope functions. Our calculation accounts for the experimental data (bending and saturation of the photoluminescence energy), but overestimates the blue-shift. This is attributed to the onset of manyboby interactions and band gap renormalisation (BGR) at high carrier densities. The values we estimate for this quantity, for the single quantum well are plotted on figure 11 as the differences between the calculated and the measured energy (open diamonds). Our data reasonably matches with results of three theoretical calculations (crosses, full and open circles) for GaAs-(Al,Ga)As.
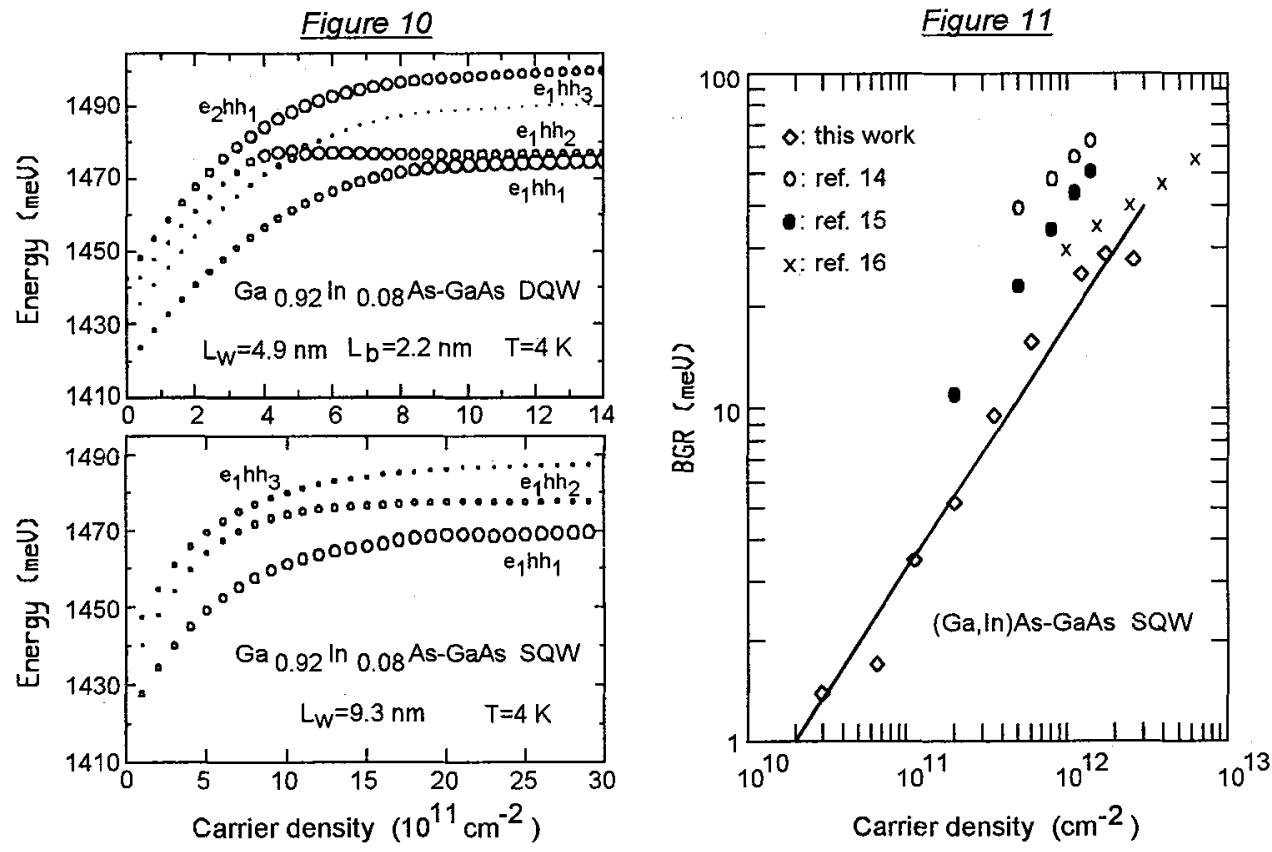

To resume this paper we plot the average hole position as a function of the carrier density on figure 12.

Figure 12

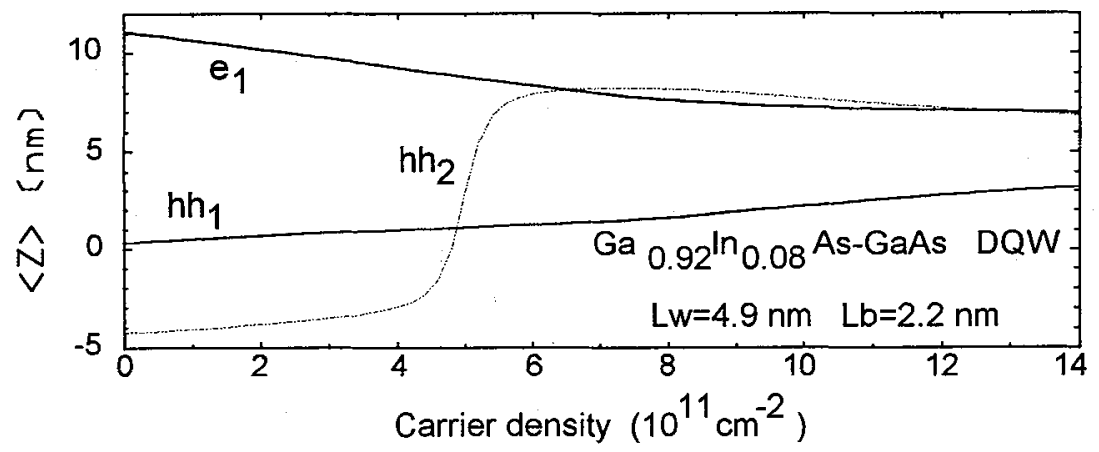


We note that the second light-hole wave functions undergoes a dramatic tunnelling at a photo carrier density of some $510^{11} \mathrm{~cm}^{-2}$. This value is commonly encountered in opto-electronic devices. Therefore we conclude that double quantum wells structures based on ( $\mathrm{Ga}$, In)As III-V semiconductors with built-in piezo-electric fjelds are promissive candidates for optoelectronic devices where advantage is taken of the photo control of the tunnelling of the minority carriers.

\section{V-Conclusion}

We have grown ( $\mathrm{Ga}, \mathrm{In})$ As-GaAs single and double quantum wells along the (001) and (111) directions. The (111) grown samples benefit from built-in internal piezoelectric field in relation with the pseudomorphic growth of the alloy to the GaAs compound. Theoretical calculation of the optical properties of the samples reveal the possibility to observe non linear optical properties in samples grown with built-in piezo electric field. This is experimentally confirmed by discriminative saturation of the photoluminescence energy at moderate photo injection between (001)- and (111)-grown samples. The non-linear properties of the samples are possible due to the long radiative lifetime of the electron-hole pairs, and are interpreted in terms of photo-induced screening of the piezoelectric field in relation with an efficient phase space filling mechanism. Moreover we demonstrate that efficient photo control of holes tunnelling may be achieved.

\section{References}

1- Stefano di Gironcoli, Stefano Baroni, and Raffaele Resta; Phys. Rev. Lett.62, 2853,(1989), Ferroelectrics, 111, 19, (1990) and references therein; Andrea Dal Corso, Raffaele Resta and Stefano Baroni, Phys. Rev. B, 47, 16252, (1993)

2- The essential aspects of their contribution to the field can be found in: D.L.Smith and C.Mailhiot, Reviews of Modern Physics, 62, 173, (1990), and in C.Mailhiot and D.L.Smith, Critical Reviews in Solid State and Materials Sciences, 16,131, (1990)

3- B.Laurich, K.Elcess, C.G.Fonstad, J.G.Beery, C.Mailhiot, and D.L.Smith, Phys. Rev. Lett 62, 649, (1989)

4- E.A.Caridi, T.Y.Chang, K.W.Goosen, and L.F.Eastman, Appl. Phys. Lett. 56, 659, (1990)., K.W.Goosen, E.A.Caridi, T.Y.Chang, J.B.Stark, D.A.B.Miller, and R.A.Morgan, Appl. Phys. Lett. 56, 715, (1990).

5- B.K.Laurich, D.L.Smith, D.E.Watkins, I.Sela, S.Subanna, and H.Kroemer, Superlattices and Microstructures, 9, 499, (1991),.I.Sela, D.E.Watkins, B.K.Laurich, and D.L.Smith, S.Subanna and H.Kroemer Appl. Phys. Lett. 58, 684, (1991).

6- B.V.Shanabrook, D.Gammon, R.Beresford, W.I.Wang, R.P.Leavitt, and D.A.Broido, in The Physics of Semiconductors, edited by E.M.Anastassakis and J.D.Joannopoulos (World Scientific Singapore, 1990), p901; B.V.Shanabrook, D.Gammon, R.Beresford, W.I.Wang, R.P.Leavitt, and D.A.Broido, Superlattices and microstructures 7, 363, (1990), G.Brozak, B.V.Shanabrook, D.Gammon, D.A.Broido, R.Beresford, and W.I.Wang, Surface Science 267, 120, (1992)

7- R.André, C.Deshayes, J.Cibert, Le Si Dang, S.Tatarenko, and K.Saminadayar, Phys. Rev. B 42, 11392, (1990), J.Cibert, R.André, C.Deshayes, Le Si Dang, 
H.Okumura, S.Tatarenko, G.Feuillet, P.H.Jouneau, R.Mallard, and K.Saminadayar Journal of Crystal Growth 117, 424, (1992)

8- M.P. Halsall, J.E.Nicholls, J.J.Davies, B.Cockayne and P.J.Wright J.Appl. Phys. $71,907,(1992)$ and references therein.

9- T.S.Moise, L.J.Guido, J. C. Beggy, T.J.Cunningham, S.Seshadri, and R.C.Barker Journal of Electronic Materials 21, 119, (1992), T.S.Moise, L.J.Guido, and R.C.Barker, J.O.White, and A.R.Kost Appl. Phys. Lett. 60, 2637, (1992).T.S.Moise, L.J.Guido, and R.C.Barker,Phys. Rev. B 47, 6758, (1993)

10- M. Lakrimi, R.W.Martin, C.Lopez, D.M.Symons, E.T.R.Chidley, R.J.Nicholas, N.J.Mason, and P.J.Walker Semicond. Sci. Technol. 8, 367, (1993)

11 - P.Boring, B.Gil, K.J.Moore, Phys. Rev. Lett. 71, 1875 (1993)

12 K.J.Moore, P.Boring, B.Gil, and K.Woodbridge, Phys. Rev. B, 48, xxxx, (1993)

13 -P.Bigenwald, B.Gil, and P.Boring, Phys. Rev B 48, 9122 ,(1993).

14 - S.Das Sarma, R. Jalabert, and S.R.Eric-Yang, Phys. Rev B, 41, 8288, (1990)

15 - P.Von Allmen, Phys. Rev B, 46, 13 345, (1992)

16 - J.C.Ryan, and T.L.Reinecke, Phys. Rev. B, 47, 9613, (1993) 